\title{
Not all choices are created equal: Task-relevant choices enhance motor learning compared to task-irrelevant choices
}

\author{
Michael J. Carter ${ }^{1,2} \cdot$ Diane M. Ste-Marie ${ }^{1}$
}

Published online: 21 February 2017

(C) Psychonomic Society, Inc. 2017

\begin{abstract}
Lewthwaite et al. (2015) reported that the learning benefits of exercising choice (i.e., their self-controlled condition) are not restricted to task-relevant features (e.g., feedback). They found that choosing one's golf ball color (Exp. 1) or choosing which of two tasks to perform at a later time plus which of two artworks to hang (Exp. 2) resulted in better retention than did being denied these same choices (i.e., yoked condition). The researchers concluded that the learning benefits derived from choice, whether irrelevant or relevant to the to-be-learned task, are predominantly motivational because choice is intrinsically rewarding and satisfies basic psychological needs. However, the absence of a group that made taskrelevant choices and the lack of psychological measures significantly weakened their conclusions. Here, we investigated how task-relevant and task-irrelevant choices affect motorskill learning. Participants practiced a spatiotemporal motor task in either a task-relevant group (choice over feedback schedule), a task-irrelevant group (choice over the color of an arm-wrap plus game selection), or a no-choice group. The results showed significantly greater learning in the taskrelevant group than in both the task-irrelevant and no-choice groups, who did not differ significantly. Critically, these learning differences were not attributed to differences in perceptions of competence or autonomy, but instead to superior error-estimation abilities. These results challenge the
\end{abstract}

Michael J. Carter

carter.michael@queensu.ca

1 School of Human Kinetics, University of Ottawa, Ottawa, Ontario, Canada

2 Centre for Neuroscience Studies, Queen's University, Kingston, Ontario, Canada perspective that motivational influences are the root cause of self-controlled learning advantages. Instead, the findings add to the growing evidence highlighting that the informational value gained from task-relevant choices makes a greater relative contribution to these advantages than motivational influences do.

Keywords Motor learning · Error estimation · Information processing $\cdot$ Autonomy $\cdot$ Competence

When learners exercise choice or control over their feedback schedule, termed self-controlled feedback, motor learning is enhanced as compared to conditions wherein this choice opportunity is denied, termed yoked feedback (for a review, see Sanli, Patterson, Bray, \& Lee, 2013). In these experiments, the self-controlled feedback group decides on a trial-to-trial basis whether they want to receive feedback regarding a justcompleted movement, whereas the participants in the yoked group are matched to a participant in the self-controlled group and replicate their feedback schedule without any choice. As such, the absolute numbers of feedback trials and their relative placement during practice are identical for both participants (see Chiviacowsky, 2014, for an alternative technique). The purpose of comparing motor performance between the selfcontrolled and yoked groups on delayed retention and transfer tests is to infer that having control over feedback is the factor contributing to any learning differences, rather than the frequency of feedback. Retention tests assess skill permanence, and transfer tests assess skill generalization (Kantak \& Winstein, 2012). Critically, self-controlled-learning advantages are not restricted to feedback and have also been shown with physical assistive devices, observation, repetition schedule, and task difficulty (for a review, see Sanli et al., 2013). 
To account for the learning advantages of self-controlled practice conditions, two (competing) explanations are generally adopted by researchers. On the one hand, some researchers have argued that having choice or control is intrinsically rewarding and satisfies the basic psychological needs of autonomy and/or competence, which in turn enhances motor learning (Chiviacowsky, 2014; Chiviacowsky, Wulf, \& Lewthwaite, 2012; Lewthwaite, Chiviacowsky, Drews, \& Wulf, 2015; Lewthwaite \& Wulf, 2012). On the other hand, the learning advantages have been suggested to be predominantly driven by the learner's ability to engage in performance-dependent strategies (e.g., error estimation) that serve to reduce the uncertainty regarding task performance (Carter, Carlsen, \& Ste-Marie, 2014; Carter, Rathwell, \& Ste-Marie, 2016; Chiviacowsky \& Wulf, 2005; Grand et al., 2015; Hansen, Pfeiffer, \& Patterson, 2011; Huang, Shadmehr, $\&$ Diedrichsen, 2008). These explanations are often referred to as the motivational-influences perspective and the information-processing perspective, respectively (see both Carter et al., 2014, and Sanli et al., 2013). To date, the learning benefits of choice for motor-skill learning have predominantly been found with choices that are relevant to learning a given motor task; however, these advantages were recently extended to choices that are irrelevant to the to-be-learned task (Lewthwaite et al., 2015).

Lewthwaite and colleagues (2015) found that allowing participants to choose their golf ball color (Exp. 1) and choosing both which of two tasks to perform at a later time and which of two artworks to hang in the laboratory (Exp. 2) resulted in significantly higher retention scores than did not being able to make these same choices. The finding that incidental choices produce beneficial effects on learning is an intriguing finding, and one that Lewthwaite et al. attributed to autonomy support (i.e., a motivational influence), because the incidental choices could not provide any information-based advantages. Although this explanation may be reasonable in terms of their data, we challenge the following conclusion made by Lewthwaite and colleagues:

That six occasions of ball color choice (Exp. 1) and two opportunities for expressing preferences (Exp. 2) were sufficient to affect motor learning suggests that the mechanism underlying previously found advantages of self-controlled and task-relevant conditions of practice may be more elemental and motivational in nature. That is, they may not pertain to creating a better fit between the learner and unfolding task-related processing, strategic, content, or other performance needs. The common denominator within the set of motor learning studies may simply be the intrinsically rewarding opportunity to exercise or anticipate control, or choice, of any kind (presumably at least somewhat meaningful and respectful in nature) - although information-processing differences may well follow motivational triggers. ( $p$. 1387)

There are two main problems with this conclusion based on their experiments. First, no motivation-related measures (e.g., autonomy, competence) were included in their design; thus, attributing the found learning differences to motivational influences such as the satisfaction of fundamental autonomy needs is unsubstantiated. Second, neither experiment included a group that made task-relevant choices; therefore, generalizing their conclusions to past self-controlled research with taskrelevant choices is unfounded. We argue that a direct comparison between task-relevant and task-irrelevant choices will provide a better lens through which to examine the relative contributions of motivational and informational influences for previously observed self-controlled learning advantages.

The purpose of the present experiment was to address the previously identified limitations of Lewthwaite et al.'s (2015) study, associated with the lack of psychological measures and a direct test of whether motor learning is differentially impacted by task-relevant and task-irrelevant choices. Participants learned a novel spatiotemporal motor task in one of three groups that differed in terms of choice: a task-relevant group, a task-irrelevant group, and a no-choice group. To test predictions from the motivational-influences perspective regarding the satisfaction of fundamental needs (Lewthwaite et al., 2015; Lewthwaite \& Wulf, 2012; Sanli et al., 2013), we administered questionnaires regarding participants' perceptions of autonomy and competence at two time points during practice. To test predictions from the information-processing perspective regarding error estimation (Carter et al., 2014; Carter \& Ste-Marie, 2016; Chiviacowsky \& Wulf, 2005), we asked participants to estimate their errors after each trial during retention and transfer. If motivational factors are the root cause of the learning benefits of choice, as Lewthwaite and colleagues (2015) argued, then no motor performance differences in retention and transfer should be found between the task-relevant and task-irrelevant groups, because the participants in both groups would have the intrinsic reward of exercising a choice, which should also satisfy basic psychological needs. Both groups should, however, demonstrate superior motor learning relative to the no-choice group. In contrast, if the root cause of the learning benefits of choice are predominantly informational, as Carter et al. (2014) argued, then task-relevant choices should yield motor performance in retention and transfer superior to that provided by taskirrelevant choices, which would still outperform the nochoice group. On the basis of Lewthwaite et al.'s (2015) conclusions, no differences in perceptions of autonomy or competence would be expected between the task-relevant and task-irrelevant groups, yet both should report significantly higher perceptions of autonomy and competence than would 
the no-choice group. Finally, we predicted that the taskrelevant group would demonstrate significantly more accurate error-estimation abilities than either the no-choice group (consistent with Carter et al., 2014; Carter \& Patterson, 2012) and the task-irrelevant group, because neither of those groups would make a choice expected to provide any taskrelated processing benefits (Lewthwaite et al., 2015).

\section{Method}

\section{Participants}

Data were collected from 54 healthy participants, who were right-handed as determined using the Edinburgh Handedness Inventory (Oldfield, 1971). All participants gave written informed consent prior to inclusion in the experiment that was approved and conducted in accordance with the ethical guidelines of the University's Research Ethics Board.

\section{Experimental groups}

Three experimental groups ( $n=18$ per group) were created: a task-relevant group $\left(M_{\text {age }}=21.56, S D=1.89 ; 9 \mathrm{M} / 9 \mathrm{~F}\right)$, a task-irrelevant group $\left(M_{\mathrm{age}}=21.22, S D=2.09 ; 10 \mathrm{M} / 8 \mathrm{~F}\right)$, and a no-choice group $\left(M_{\text {age }}=21.83, S D=2.33 ; 9 \mathrm{M} / 9 \mathrm{~F}\right)$. The participants in the task-relevant group were permitted choice over their feedback schedule throughout practice; however, they were restricted to 20 feedback trials (33\%) and the exception that all 20 requests had to be used. If the number of remaining requests matched the remaining number of practice trials, these trials would default to feedback trials. ${ }^{1}$ We restricted the number of feedback requests so that all participants, within and between groups, would practice with the same frequency of feedback. Restricting the number of feedback requests is not uncommon in such research (e.g., Carter et al., 2014; Carter \& Ste-Marie, 2016; Chiviacowsky, 2014; Chiviacowsky \& Wulf, 2005; Chiviacowsky, Wulf, \& Lewthwaite, 2012) and has also resulted in more desirable learning outcomes than occur with an unrestricted number of feedback opportunities during practice (Hansen et al., 2011).

The participants in the task-irrelevant group were provided a choice between which of two video games to play at the end of data collection on Day 2 (3D Pong or Duck! Jump!) and which color arm-wrap (yellow, beige, or blue) to have placed around the arm used to learn the motor task. Both decisions were made immediately prior to starting the first practice block, and these choices could not be modified after they had been made. Thus, a main distinction between the taskrelevant and task-irrelevant groups, other than what their

\footnotetext{
${ }^{1}$ This only occurred for three participants in the task-relevant group.
}

choice reflected, was that the former exercised choice during practice, whereas the latter exercised choice before practice.

Finally, the participants in the no-choice group were not permitted any choices. Instead, the three arm-wrap color options were made visible, and the experimenter chose the color. As for the game, no-choice participants were told that there were two options, 3D Pong or Duck! Jump!, of games that could be played the following day, but that the choice had already been made for them. All participants in the taskirrelevant and no-choice groups completed one round of the game after completing the delayed retention and transfer tests, but performance on the video game was not recorded (consistent with Lewthwaite et al., 2015). The same procedure regarding the arm-wrap with the no-choice group was also used with the task-relevant group. Importantly, the arm-wrap provided no benefit for learning the motor task, and the color never changed during practice. All participants were told that the purpose of the arm-wrap was to keep their arm in the correct position within the armrest of the manipulandum.

The participants in the task-irrelevant and no-choice groups were yoked to the feedback schedule created by a participant in the task-relevant group and were told that they would receive feedback on a predetermined schedule. Thus, all participants, independent of group, practiced with identical feedback schedules in terms of frequency (33\%) and relative placement throughout practice. Because the feedback schedules of the task-relevant participants were needed for the other two groups, the first 18 participants were placed into the taskrelevant group, whereas the last 36 participants were randomly assigned to either the task-irrelevant or the no-choice group. A summary of the experimental groups and the respective manipulations can be found in Table 1 .

\section{Task and apparatus}

The task required participants to perform two rapid extensionflexion reversals about the elbow of their nondominant arm to produce a target waveform trajectory as accurately as possible (Fig. 1a). The spatiotemporal pattern was created by summing two sine waves: $y(t)=42 \sin (\pi t-0.3)+23 \sin (3 \pi t+0.4)$. The waveform task was adopted from Goh, Sullivan, Gordon, Wulf, and Winstein (2012), and we have successfully used this task in a self-controlled feedback paradigm (Carter \& Ste-Marie, 2016). The overall movement time goal was $900 \mathrm{~ms}$ during practice and retention. For transfer, the same waveform trajectory was used, but the overall goal movement time was $1,150 \mathrm{~ms}$.

During all experimental phases, the participants sat facing a 24-in. computer monitor with their left forearm in an armrest, such that their elbow was bent at $90^{\circ}$. The armrest was attached to a custom-made manipulandum affixed to an axis that restricted movement to the horizontal plane. Arm-wrap was placed around the participants' left forearm and armrest 
Table 1 Summary of the experimental groups and the aspects that participants had choice over and those that were imposed on them

\begin{tabular}{lll}
\hline Group & Choice feature & Imposed feature \\
\hline Task-relevant group & Feedback schedule & Arm-wrap color \\
Task-irrelevant group & Arm-wrap color + Game to play & Feedback schedule \\
No-choice group & Nothing & Feedback schedule + Arm-wrap color + Game to play \\
\hline
\end{tabular}

These manipulations were only present during the practice phase

(see the Experimental groups section for greater details) and was only present during the practice phase. Participants grasped an adjustable handle to ensure the central axis of rotation was about the elbow joint and vision of the entire arm was occluded. A linear potentiometer powered by a $5-\mathrm{V}$ direct-current power supply attached to the central axis of each handle provided position data that were sampled at $1000 \mathrm{~Hz}$ for the duration of each motor response using analog to digital hardware (National Instruments Inc. PCIe-6321). A custom LabVIEW (National Instruments Inc.) program was created that controlled the timing of all experimental stimuli for each trial and recorded and stored all data for offline analysis.

\section{Procedure}

All participants had a series of instructions read to them as they followed along on the monitor in front of them prior to completing each phase of the experiment. These instructions described the motor task and its associated movement time goal (900 or 1,150 ms, depending on experimental phase); however, no explicit information regarding the amplitudes of the two reversal points were provided. Three example feedback displays were shown to all participants to ensure participants understood how to interpret feedback whenever it was provided. The instructions also consisted of group specific information regarding their choice manipulation as outlined in the Experimental groups section.

Trials began with the target waveform displayed $(2,000 \mathrm{~ms})$, followed by a visual "Get Ready" and a visual "Go" signal (1,000 ms apart). Participants could start their movement when ready following the "Go" signal and the monitor was black during the movement period. Upon movement completion, a 3,000-ms feedback delay interval occurred that was followed by the feedback decision prompt for the task-relevant group. On trials that feedback was chosen (i.e., task-relevant group) or imposed (i.e., task-irrelevant and nochoice groups) it was displayed for 3,000 ms and consisted of the participant's waveform trace superimposed on the target waveform, plus their actual movement time in milliseconds. On non-feedback trials, a black screen was displayed for 3,000 ms (Fig. 1b).

The practice phase consisted of six blocks of ten trials, and after Blocks 1 and 6, participants answered questions on a 7 point Likert scale to assess their perceived competence and autonomy (Table 2). The perceived competence questions related to task performance and were taken from the Intrinsic Motivation Inventory (McAuley, Duncan, \& Tammen, 1989). Because it is argued from the motivational perspective that
A

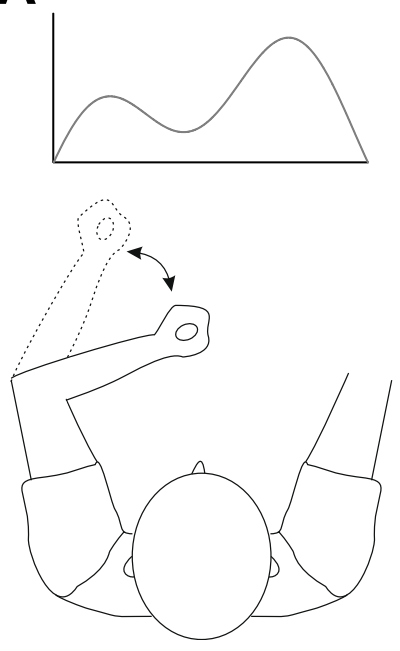

Fig. 1 a Schematic representation of the goal waveform, which was reproduced by participants with two extension-flexion reversals. b An outline of the sequence of events during the practice phase for the task-

B

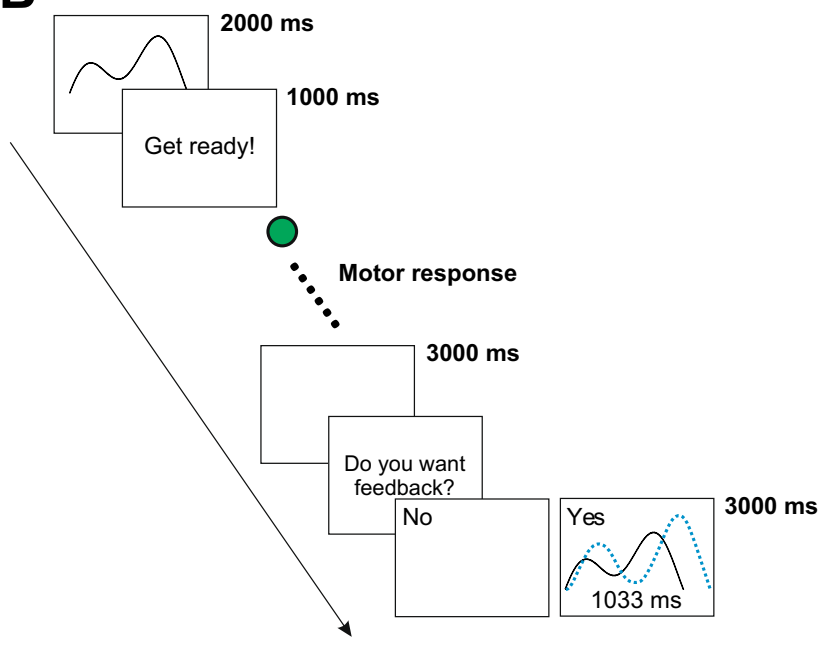

relevant group. The only difference for the task-irrelevant and no-choice groups was that no feedback prompt occurred. The feedback prompt was also not present during the retention and transfer phases of the experiment 
Table 2 The 14 questions that were given to participants after Blocks 1 and 6 during the practice phase

Question

Construct

1. I think I am pretty good at this waveform matching task.

Competence

2. During practice, I feel free to make choices and express my opinions.

Autonomy (D)

3. I think I did pretty well at this waveform matching task compared to others.

Competence

4. I feel controlled and pressured during practice.

Autonomy (D)

5. After working at this waveform matching task for a while, I felt pretty competent.

Competence

6. Before practice, I feel like my choices and opinions were taken into consideration.

Autonomy (B)

7. I am satisfied with my performance on this waveform matching task.

Competence

8. There is not much opportunity for me to exercise choices during practice.

Autonomy (D)

9. I was pretty skilled at this waveform matching task.

Competence

10. Before practice, I felt free to make choices and express my opinions.

Autonomy (B)

11. The waveform matching task is an activity that I couldn't do very well.

Competence

12. I felt controlled and pressured before practice.

Autonomy (B)

13. During practice, I feel like my choices and opinions are taken into consideration.

Autonomy (D)

14. There was not much opportunity for me to exercise choices before practice.

Autonomy (B)

These questions were answered on a 7-point Likert scale. The construct column refers to the psychological construct that an item assessed. Competence $=$ Perceived competence scale from the IMI; Autonomy (B) = Perceived autonomy before practice started; Autonomy (D) = Perceived autonomy during practice

providing choice to learners satisfies a fundamental need for autonomy (Lewthwaite et al., 2015), it is critical that the questions used to assess perceived autonomy reflect one's experimental group and not the motor task (for a discussion, see Carter et al., 2014). For this reason, the perceived choice subscale of the IMI was not used and we created our own questions instead. Since the task-relevant and task-irrelevant groups exercised their choices at different times (during practice vs. before practice, respectively), this difference was reflected in the questions for perceived autonomy. The retention and transfer tests consisted of one block of ten nofeedback trials and after each trial participants were asked to estimate their error in milliseconds with respect to the overall movement time goal. Error estimations were verbalized by the participant and entered in an Excel spreadsheet for later analysis. The retention and transfer tests were conducted approximately $24 \mathrm{~h}$ after the practice phase.

\section{Data analysis}

Overall performance accuracy relative to the goal waveform was assessed using root mean square error (RMSE), which is the mean difference between the goal waveform and the participant's movement trajectory calculated over the participant's actual movement time. RMSE was calculated after synchronizing the onset of the target waveform with the participant's response. RMSE is sensitive to errors in both spatial and temporal domains and captures both variability and bias of the performed motor response (Schmidt \& Lee, 2011). The RMSE values for individual trials were averaged to generate an overall RMSE score for each block of ten trials. For the retention and transfer tests, we determined the total estimation error (Bruechert, Lai, \& Shea, 2003), which was calculated using the formula

$E E=\sqrt{ }\left(C E^{2}+V E^{2}\right)$,

where constant error (CE) captured estimation bias (estimated - actual) and variable error (VE) was the standard deviation of these errors. The values for estimation error (EE) for individual trials were averaged to generate an overall EE score for both retention and transfer. Perceived competence and perceived autonomy scores were averaged across the subset of questions pertaining to these psychological constructs. Differences with a probability of $<.05$ were considered significant and partial eta-squared $\left(\eta_{\mathrm{p}}{ }^{2}\right)$ is reported as an estimate of effect size. Post-hoc analyses were performed using Tukey's HSD or Holm-Bonferroni procedures and in cases in which the assumption of sphericity was violated, GreenhouseGeisser-adjusted $p$ values are reported.

\section{Results}

\section{RMSE}

Practice Mean RMSE scores for practice were analyzed in a 3 (Group: task-relevant, task-irrelevant, no-choice) $\times 6$ (Block) mixed-model analysis of variance (ANOVA) with repeated measures on block. Overall, performance accuracy increased in all experimental groups (Fig. 2), which was supported by a significant block main effect, $F(5,255)=19.44, p<.001, \eta_{\mathrm{p}}{ }^{2}$ 


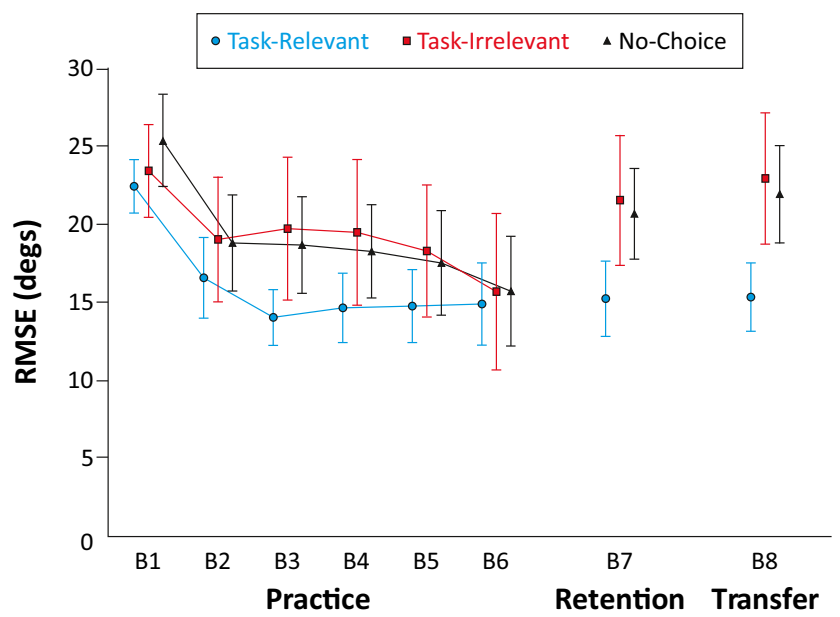

Fig. 2 Mean RMSEs for the practice phase (B1-B6), the retention phase (B7), and the transfer phase (B8). Each block consisted of ten trials, and feedback was available only during practice. The task-relevant group (circles) performed with significantly greater accuracy during retention and transfer than did the task-irrelevant (squares) and no-choice (triangles) groups. Data points are offset to increase the legibility of the error bars. Error bars represent $95 \%$ confidence intervals. For the color version of this figure, the reader is referred to the online version of this article

$=.28$. Tukey's post-hoc comparisons revealed that more errors occurred in Block 1 than in all the other blocks. Importantly, no significant group differences were noted in Block 1 or Block 6. The main effect of group, $F(2,51)=1.95, p=.15$, and the Group $\times$ Block interaction, $F(10,255)<1, p=.59$, were not significant.

Retention and transfer The mean RMSE scores for retention and transfer were analyzed in separate one-way ANOVAs. The task-relevant group had the lowest errors in retention and transfer (Fig. 2), which were supported by significant main effects of group in both retention, $F(2,51)=4.09, p=$ $.023, \eta_{\mathrm{p}}{ }^{2}=.14$, and transfer, $F(2,51)=6.30, p=.004, \eta_{\mathrm{p}}{ }^{2}=$ .20. Holm-Bonferroni comparisons showed that during retention, the task-relevant group was significantly more accurate than both the task-irrelevant and no-choice groups, who did not differ significantly from each other. Holm-Bonferroni comparisons revealed the same pattern of results for transfer.

\section{Perceived competence}

Mean scores for perceived competence (Fig. 3a) were analyzed in a 3 (Group) $\times 2$ (Time) ANOVA with repeated measures on time. Perceived competence scores increased from Time 1 (Block 1) to Time 2 (Block 6), which was supported by a significant main effect of time, $F(1,51)=72.55, p<.001$, $\eta_{\mathrm{p}}{ }^{2}=.59$. Neither the main effect of group, $F(2,51)<1, p=$ .782 , nor the Group $\times$ Time interaction, $F(2,51)<1, p=.832$, was significant.
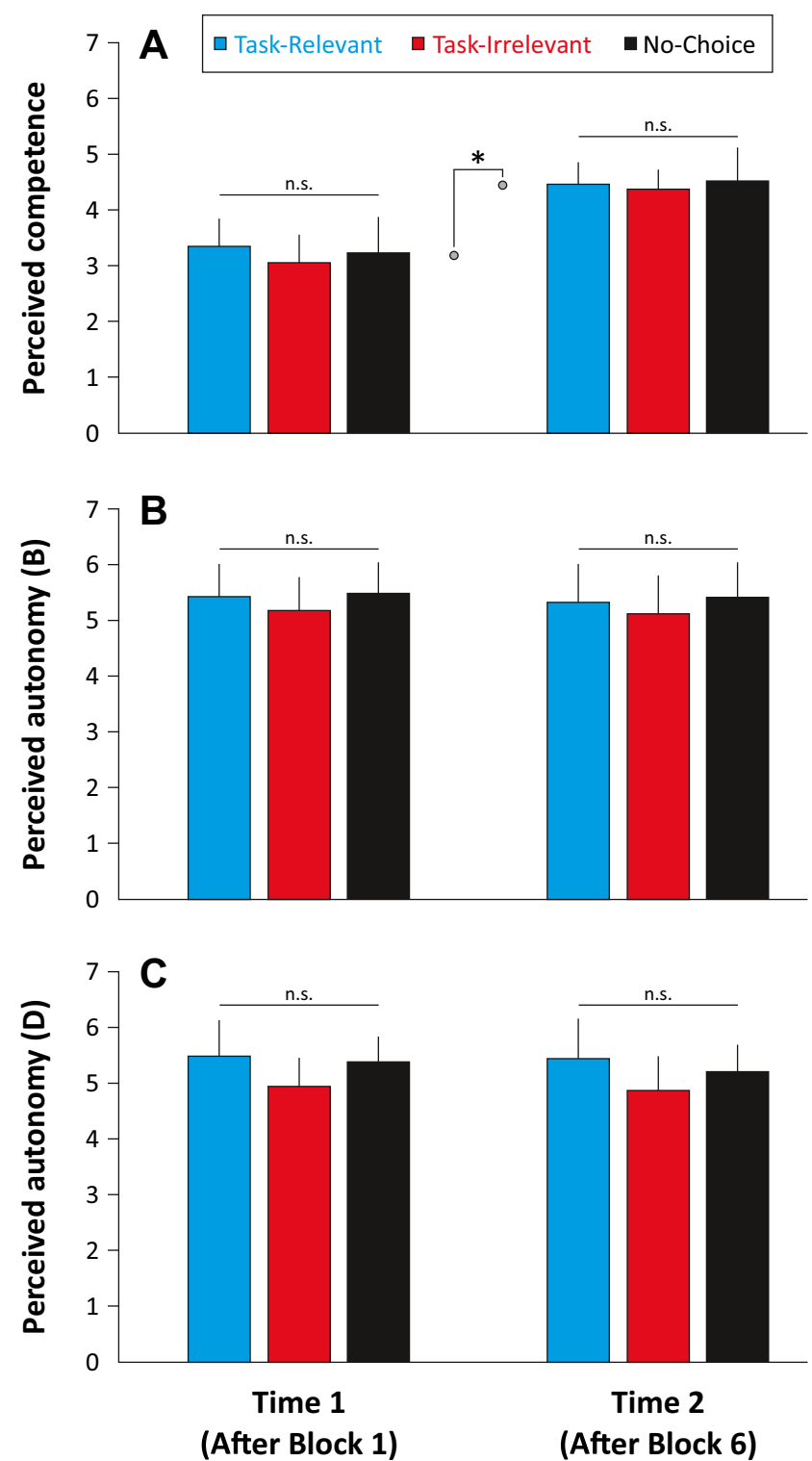

Fig. 3 Mean competence and autonomy scores for the task-relevant (left bar in each set), task-irrelevant (middle bars), and no-choice (right bars) groups at Times 1 and 2, which corresponded to after Blocks 1 and 6 , respectively. There were no significant group differences for perceived competence (a); however, perceived competence significantly increased from the start to the end of practice (gray circles represent the grand means for Time 1 and Time 2). $\mathbf{b}$ and $\mathbf{c}$ Perceived autonomy scores for the questions that asked participants about how well their need for autonomy was satisfied before (b) and during (c) the practice phase. No significant group differences were found. Errors bars represent 95\% confidence intervals. For the color version of this figure, the reader is referred to the online version of this article

\section{Perceived autonomy}

Before practice The mean scores for perceived autonomy (Fig. 3b) were analyzed in a 3 (Group) $\times 2$ (Time) ANOVA with repeated measures on time. The main effects of group, $F(2,51)<1, p=.776$, and time, $F(1,51)<1, p=.458$, as well 
as the interaction of these two factors, $F(2,51)<1, p=.964$, failed to reach statistical significance.

During practice The mean scores for perceived autonomy (Fig. 3c) were analyzed in a 3 (Group) $\times 2$ (Time) ANOVA with repeated measures on time. The main effects of group, $F(2,51)=1.01, p=.371$, and time, $F(1,51)<1, p=.354$, as well as the Group $\times$ Time interaction, $F(2,51)<1, p=.864$, were not significant.

\section{Total EE}

Mean EE scores (Fig. 4) for retention and transfer were analyzed in separate one-way ANOVAs. The task-relevant group had lower EE scores in retention and transfer than did the taskirrelevant and no-choice groups. The main effect of group approached significance during retention, $F(2,51)=3.02, p$ $=.058, \eta_{\mathrm{p}}^{2}=.11$; however, it was significant during transfer, $F(2,51)=7.57, p=.001, \eta_{\mathrm{p}}{ }^{2}=.23$. Holm-Bonferroni posthoc tests revealed that the task-relevant group had significantly less EE than did the task-irrelevant and no-choice groups, who did not differ significantly.

\section{Discussion}

Motor-learning experiments have consistently provided evidence for the effectiveness of self-controlled practice conditions (i.e., exercising choice over a specific feature) as a means for enhancing skill retention and/or transfer. These learning benefits have predominantly been found with choices that are relevant to the motor task being learned, and only recently

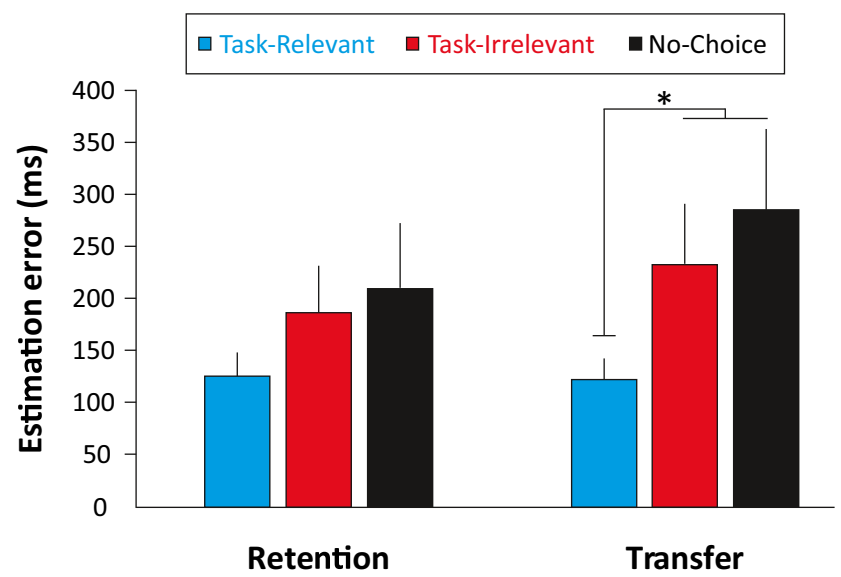

Fig. 4 Mean estimation error scores for the task-relevant (left bar in each panel), task-irrelevant (middle bar), and no-choice (right bar) groups during retention and transfer. The task-relevant group had less estimation error than the task-irrelevant and no-choice groups, but this difference was significant only in transfer $(p=.001)$, and approached significance in retention $(p=.058)$. Error bars represent $95 \%$ confidence intervals. For the color version of this figure, the reader is referred to the online version of this article were extended to choices irrelevant to learning a given motor task (Lewthwaite et al., 2015). Lewthwaite et al. concluded that the root cause of the learning advantages associated with both task-relevant and task-irrelevant choices are motivational in nature and attributable to the satisfaction of basic psychological needs (e.g., autonomy and competence). However, some methodological limitations of their experiment weaken their conclusions. Here, we addressed these limitations by including a task-relevant group and measures associated with the motivational explanation, namely, perceptions of competence and autonomy (e.g., Sanli et al., 2013).

The data showed a distinct retention and transfer advantage on the waveform task for task-relevant choices than for taskirrelevant choices and not being afforded any choices (Fig. 2). Although we found no group differences regarding perceptions of competence or autonomy (Fig. 3), the task-relevant group was significantly more accurate at error estimation in transfer than were the task-irrelevant and no-choice groups, who did not differ significantly (Fig. 4). These combined findings impact current theoretical discussions pertaining to the mechanisms that underlie the facilitative effects of choice on motor learning. Currently, the dominant view favors motivational factors as the primary mechanism as it is argued that exercising choice is not only intrinsically rewarding but also supports the learner's fundamental needs for autonomy and/or competence, which in turn enhance intrinsic motivation and motor learning (Chiviacowsky, 2014; Chiviacowsky, Wulf, \& Lewthwaite, 2012; Lewthwaite \& Wulf, 2010, 2012; Sanli et al., 2013). However, our lack of significant differences between groups regarding perceptions of autonomy and competence, despite the enhanced learning of the task-relevant group relative to the task-irrelevant and no-choice groups, who did not differ significantly, contests the proposition that motivational factors are the root cause of self-controlled learning advantages (e.g., Lewthwaite et al., 2015).

Although the notion that choice benefits motor learning because it is autonomy-supportive is often concluded (e.g., Chiviacowsky, 2014; Lewthwaite et al., 2015), the lack of autonomy support measures in this area of motor learning research has been especially problematic (Chiviacowsky, 2014; Chiviacowsky, Wulf, \& Lewthwaite, 2012; e.g., Chiviacowsky, Wulf, Lewthwaite, \& Campos, 2012; Chiviacowsky, Wulf, Machado, \& Rydberg, 2012; Kaefer, Chiviacowsky, Meira, \& Tani, 2014; Lewthwaite et al., 2015). To our knowledge, we are the first to actually test perceived autonomy as a function of one's choice condition (cf. Ste-Marie, Vertes, Law, \& Rymal, 2013, who examined perceived choice with respect to executing the motor task via the IMI). Interestingly, we found no support that exercising choice, whether relevant or irrelevant to the to-be-learned task, is more autonomy-supportive than not exercising choice. This finding is consistent with Ste-Marie et al. (2013), who found no differences in perceived competence between their self- 
controlled and yoked groups regarding the double-mini trampoline task, despite finding the typical self-controlled learning advantages. Although we recognize the weakness associated with the lack of validation of the autonomy-support measure, the statements used have strong face validity (Table 2) and thus, our contention is that the data still provides initial insight that autonomy support does not adequately account for the observed learning benefits of task-relevant choices over both task-irrelevant choices and being denied choice. Similarly, no significant group differences were noted for perceived competence with the task at either time point. Although this finding is inconsistent with the motivation explanation (Sanli et al., 2013; Wulf \& Lewthwaite, 2016), it is consistent with past research (Chiviacowsky, Wulf, \& Lewthwaite, 2012; Grand et al., 2015). Grand et al., for example, found enhanced learning in a self-controlled feedback group as compared to a yoked feedback group, despite the two groups not differing significantly in terms of their perceived competence with the motor task. $^{2}$ Our data did show a significant increase in perceived competence over time that mirrored the significant increase in physical performance; thus, had group differences existed, the perceived competence measure should have been sensitive to them.

Given the absence of supporting evidence for autonomysupport and perceived competence, alternative explanations to the motivational perspective need to be considered to better understand the advantages associated with choice in motor learning. Accordingly, we (Carter et al., 2014; Carter et al., 2016; Carter \& Ste-Marie, 2016; Ste-Marie, Carter, Law, Vertes, \& Smith, 2016) continue to assert that choice over one's feedback schedule encourages information-processing activities such as error estimation that ultimately reduce uncertainty regarding task-performance that increases the relative permanence and/or generalizability of the acquired motor skill (also see Chiviacowsky \& Wulf, 2005; Fairbrother, Laughlin, \& Nguyen, 2012; Grand et al., 2015). Although it was expected that the task-relevant group would demonstrate greater error-estimation abilities in both retention and transfer on the basis of previous work (Carter et al., 2014; Carter \& Patterson, 2012), the data showed that the task-relevant group was numerically, but not statistically, more accurate in retention. The differences, however, were significant in transfer with the task-relevant group being superior to both the taskirrelevant and no-choice groups, whereas both the taskirrelevant and no-choice groups were not significantly different on either test. It has been suggested in past self-controlled research that retention tests are less sensitive measures of learning than transfer tests (Chiviacowsky \& Wulf, 2002, 2005) and that transfer-specific learning advantages of having task-relevant choices are likely due to error-estimation

\footnotetext{
${ }^{2}$ Grand et al. (2015) also found no significant differences on the IMI subscales of interest/enjoyment or effort/importance.
}

processes that result in participants being able to more effectively adapt or scale performance when presented with novel task requirements (Aiken, Fairbrother, \& Post, 2012; Grand et al., 2015). This explanation seems plausible for the taskrelevant group's enhanced error-estimation in transfer given that participants would have had to rely on independent error detection and correction processes to accurately scale their performance to a novel MT goal $(1,150 \mathrm{~ms})$. It is puzzling though as to why this enhanced error detection and correction mechanism would not also produce a significant benefit in retention given subjective error-estimation must also be relied on for trial-to-trial changes in performance as feedback (i.e., knowledge of results [KR]) was not available in either retention or transfer. It should be noted, however, that the Group effect in retention approached significance with a medium effect size ( $p=.058, \eta_{\mathrm{p}}{ }^{2}=.11$; Gray \& Kinnear, 2012). Additionally, Fig. 3 also reveals that the task-relevant group's mean estimation error in retention and transfer were similar (a difference of $3.66 \mathrm{~ms}$ ), whereas estimation error increased in transfer from retention for both the task-irrelevant $(+47.17 \mathrm{~ms})$ and no-choice $(+74.64 \mathrm{~ms})$ groups. Taking these combined factors into consideration, along with the conclusions of Aiken et al. (2012) and Grand et al. (2015), we argue that Fig. 3 provides some evidence that an overall more reliable and accurate error detection and correction mechanism was developed by participants in the task-relevant group than for all other groups.

Chiviacowsky and Wulf (2005) were the first to suggest error-estimation processes as a key determinant of the learning benefits of choice in motor learning; however, they did not have a measure to support this proposition. Since then, evidence for a greater relative contribution of informational factors including error-estimation (Carter et al., 2014; Carter \& Patterson, 2012), and more recently, augmented feedback processing (Grand et al., 2015) has accumulated in recent years. Further support comes from the finding that interposing the feedback-delay interval, which is when error-estimation processes are thought to naturally occur (Swinnen, 1988), with a secondary task eliminates the learning advantages of selfcontrolled feedback schedules (Carter \& Ste-Marie, 2016). Similarly, these learning benefits are also eliminated if outcome feedback (i.e., KR) is redundant with task-intrinsic feedback and would therefore serve little-to-no informational value for the development of error-estimation abilities (Barros, Tran, Aisner, \& Salvadora, 2015). Collectively, these findings highlight the prominent role of informational factors for explaining self-controlled learning advantages; however, we encourage others to replicate these finding with not only selfcontrolled feedback manipulations, but also with choice over other task-relevant variables (e.g., observation, repetition schedule).

Although we replicated the standard learning benefit of choice, we failed to replicate the findings of Lewthwaite 
et al. (2015). Although we used a different motor task in the present experiment, the incidental choices afforded to the taskirrelevant group and the yoking procedure for these choices with the no-choice group were consistent with Lewthwaite et al.'s methods. Thus, it is unclear why we failed to replicate their findings with a different motor task given that a metaanalysis showed that the robust learning benefits associated with self-controlled conditions do not depend on task type (e.g., lab-based versus applied; McKay, Carter, \& Ste-Marie, 2014). The issue of replication has been widely discussed as of late (Simons, 2014) and recently with respect to motorlearning research (Lohse, Buchanan, \& Miller, 2016); thus, we encourage other research groups to test the effectiveness of task-irrelevant choices as replication attempts from different research laboratories is necessary to determine whether the incidental choice effect is a robust phenomenon for motor learning (Open Science Collaboration, 2015).

A limitation of the present experiment is that the taskrelevant group exercised choice more times than the taskirrelevant group (20 vs. 2 times, respectively). As such, it could be argued that the found learning differences between these two groups resulted from the amount of choice rather than the relevancy of the choice. We refute this possibility as it has been shown that reducing the number of choice opportunities by $50 \%$ (90 vs. 45 opportunities), while holding the amount of practice constant, did not compromise the learning benefits of self-controlled feedback schedules (Patterson, Carter, \& Sanli, 2011). Thus, the amount of choice does not seem to be a determining factor for the learning advantages associated with choice. We are therefore confident that the learning differences between the task-relevant and taskirrelevant groups are the result of task-relevant choices simply being more effective for learning.

A final consideration regarding not only the present experiment, but also the extant self-controlled motor learning literature is the fact that the benefits of choice did not emerge during practice, and instead only manifest on delayed retention and/or transfer tests. This consistent trend in the literature warrants investigation and it may be fruitful for future experimentation to consider motor memory consolidation processes (Kantak \& Winstein, 2012; Robertson, 2009; Robertson, Pascual-Leone, \& Miall, 2004) and/or transfer-appropriate processing (Bransford, Franks, Morris, \& Stein, 1979; Lee, 1988; Morris, Bransford, \& Franks, 1977). These avenues of research could serve as complementary or alternative explanations for the motor learning benefits associated with choice (see Andrieux, Boutin, \& Thon, 2015; Carter et al., 2014, for discussions).

In sum, we have provided the first evidence that taskrelevant and task-irrelevant choices are not equally beneficial for motor skill learning. From a practical standpoint, the data strongly suggest that in situations (e.g., rehabilitation, vocation, and recreation) in which learners may be provided with some degree of choice while learning or relearning a motor skill, these choices should be exercised over a feature that is directly related to the task they are attempting to learn (e.g., feedback, repetition schedule, task difficulty, and modeled demonstrations). At the theoretical level, our questionnaire data challenges autonomy-support and perceived competence, and any resulting increases in motivation as the root cause for the learning advantages of choice (Lewthwaite et al., 2015; Sanli et al., 2013). Instead, the data suggests that the mechanisms underlying the learning advantages of self-controlled practice conditions are strongly influenced by informational factors and are associated with task-related processing activities, such as error-estimation. Although self-controlled (i.e., choice) practice conditions may also benefit learning through motivational influences, such influences are far more modest in nature than are information-based factors (e.g., Grand et al., 2015; Ste-Marie et al., 2016).

Author note The authors thank Abdo Elnakouri and Zachary Yantha for their help with participant recruitment and data collection.

\section{References}

Aiken, C. A., Fairbrother, J. T., \& Post, P. G. (2012). The effects of selfcontrolled video feedback on the learning of the basketball set shot. Frontiers in Psychology, 3, 338. doi:10.3389/fpsyg.2012.00338

Andrieux, M., Boutin, A., \& Thon, B. (2015). Self-control of task difficulty during early practice promotes motor skill learning. Journal of Motor Behavior, 48, 57-65. doi:10.1080/00222895.2015.1037879

Barros, J. A. C., Tran, A. Q., Aisner, T., \& Salvadora, L. T. (2015). Effects of self-controlled feedback on the acquisition of a balance task. Journal of Sport and Exercise Psychology, 31(Suppl), S28.

Bransford, J. D., Franks, J. J., Morris, C. D., \& Stein, B. S. (1979). Some general constraints on learning and memory research. In L. S. Cermak \& F. I. M. Craik (Eds.), Levels of processing in human memory (pp. 331-354). Hillsdale: Erlbaum.

Bruechert, L., Lai, Q., \& Shea, C. H. (2003). Reduced knowledge of results frequency enhances error detection. Research Quarterly for Exercise and Sport, 74, 467-472.

Carter, M. J., Carlsen, A. N., \& Ste-Marie, D. M. (2014). Self-controlled feedback is effective if it is based on the learner's performance: A replication and extension of Chiviacowsky and Wulf (2005). Frontiers in Psychology, 5, 1325. doi:10.3389/fpsyg.2014.01325

Carter, M. J., \& Patterson, J. T. (2012). Self-controlled knowledge of results: Age-related differences in motor learning, strategies, and error detection. Human Movement Science, 31, 1459-1472. doi: 10.1016/j.humov.2012.07.008

Carter, M. J., Rathwell, S., \& Ste-Marie, D. M. (2016). Motor skill retention is modulated by strategy choice during self-controlled knowledge of results schedules. Journal of Motor Learning and Development, 4, 100-115. doi:10.1123/jmld.2015-0023

Carter, M. J., \& Ste-Marie, D. M. (2016). An interpolated activity during the knowledge-of-results delay interval eliminates the learning advantages of self-controlled feedback schedules. Psychological Research. Advance online publication. doi:10.1007/s00426-016$0757-2$

Chiviacowsky, S. (2014). Self-controlled practice: Autonomy protects perceptions of competence and enhances motor learning. 
Psychology of Sport and Exercise, 15, 505-510. doi:10.1016/j. psychsport.2014.05.003

Chiviacowsky, S., \& Wulf, G. (2002). Self-controlled feedback: Does it enhance learning because performers get feedback when they need it? Research Quarterly for Exercise and Sport, 73, 408-415.

Chiviacowsky, S., \& Wulf, G. (2005). Self-controlled feedback is effective if it is based on the learner's performance. Research Quarterly for Exercise and Sport, 76, 42-48.

Chiviacowsky, S., Wulf, G., Lewthwaite, R., \& Campos, T. (2012). Motor learning benefits of self-controlled practice in persons with Parkinson's disease. Gait and Posture, 35, 601-605. doi:10.1016/j. gaitpost.2011.12.003

Chiviacowsky, S., Wulf, G., Machado, C., \& Rydberg, N. (2012). Selfcontrolled feedback enhances learning in adults with Down syndrome. Revista Brasileira de Fisioterapia, 16, 191-196.

Chiviacowsky, S., Wulf, G., \& Lewthwaite, R. (2012). Self-controlled learning: The importance of protecting perceptions of competence. Frontiers in Psychology, 3, 458. doi:10.3389/Fpsyg.2012.00458

Fairbrother, J. T., Laughlin, D. D., \& Nguyen, T. V. (2012). Selfcontrolled feedback facilitates motor learning in both high and low activity individuals. Frontiers in Psychology, 3, 323. doi:10.3389/ fpsyg.2012.00323

Goh, H. T., Sullivan, K. J., Gordon, J., Wulf, G., \& Winstein, C. J. (2012). Dual-task practice enhances motor learning: A preliminary investigation. Experimental Brain Research, 222, 201-210. doi:10.1007/ s00221-012-3206-5

Grand, K. F., Bruzi, A. T., Dyke, F. B., Godwin, M. M., Leiker, A. M., Thompson, A. G., ... Miller, M. W. (2015). Why self-controlled feedback enhances motor learning: Answers from electroencephalography and indices of motivation. Human Movement Science, 43, 23-32. doi:10.1016/j.humov.2015.06.013

Gray, C., \& Kinnear, P. (2012). IBM SPSS Statistics 19 made simple. New York: Psychology Press.

Hansen, S., Pfeiffer, J., \& Patterson, J. T. (2011). Self-control of feedback during motor learning: Accounting for the absolute amount of feedback using a yoked group with self-control over feedback. Journal of Motor Behavior, 43, 113-119. doi:10.1080/00222895.2010. 548421

Huang, V. S., Shadmehr, R., \& Diedrichsen, J. (2008). Active learning: Learning a motor skill without a coach. Journal of Neurophysiology, 100, 879-887. doi:10.1152/jn.01095.2007

Kaefer, A., Chiviacowsky, S., Meira, C. D., \& Tani, G. (2014). Selfcontrolled practice enhances motor learning in introverts and extroverts. Research Quarterly for Exercise and Sport, 85, 226-233. doi: 10.1080/02701367.2014.893051

Kantak, S. S., \& Winstein, C. J. (2012). Learning-performance distinction and memory processes for motor skills: A focused review and perspective. Behavioural Brain Research, 228, 219-231. doi:10.1016/j. bbr.2011.11.028

Lee, T. D. (1988). Transfer-appropriate processing: A framework for conceptualizing practice effects in motor learning. In O. G. Meijer $\&$ K. Roth (Eds.), Complex movement behaviour: The motor-action controversy (pp. 201-215). Amsterdam: Elsevier Science.

Lewthwaite, R., Chiviacowsky, S., Drews, R., \& Wulf, G. (2015). Choose to move: The motivational impact of autonomy support on motor learning. Psychonomic Bulletin \& Review, 22, 1383-1388. doi:10. 3758/s13423-015-0814-7

Lewthwaite, R., \& Wulf, G. (2010). Grand challenge for movement science and sport psychology: Embracing the social-cognitive-affective-motor nature of motor behavior. Frontiers in Psychology, 1, 42. doi:10.3389/fpsyg.2010.00042
Lewthwaite, R., \& Wulf, G. (2012). Motor learning through a motivational lens. In N. J. Hodges \& A. M. Williams (Eds.), Skill acquisition in sport: Research, theory, and practice (2nd ed., pp. 173-191). London: Routledge.

Lohse, K., Buchanan, T., \& Miller, M. (2016). Underpowered and overworked: Problems with data analysis in motor learning studies. Journal of Motor Learning and Development, 4, 37-58. doi:10. 1123/jmld.2015-0010

McAuley, E., Duncan, T., \& Tammen, V. V. (1989). Psychometric properties of the Intrinsic Motivation Inventory in a competitive sport setting: A confirmatory factor-analysis. Research Quarterly for Exercise and Sport, 60, 48-58.

McKay, B., Carter, M. J., \& Ste-Marie, D. M. (2014). Self-controlled learning: A meta-analysis. Journal of Sport and Exercise Psychology, 36(Suppl), S43.

Morris, C. D., Bransford, J. D., \& Franks, J. J. (1977). Levels of processing versus transfer appropriate processing. Journal of Verbal Learning and Verbal Behavior, 16, 519-533. doi:10.1016/S00225371(77)80016-9

Oldfield, R. C. (1971). The Assessment and analysis of handedness: The Edinburgh Inventory. Neuropsychologia, 9, 97-113. doi:10.1016/ 0028-393290067-4

Open Science Collaboration. (2015). Estimating the reproducibility of psychological science. Science, 349, aac4716-aac4716. doi:10. 1126/science.aac4716

Patterson, J. T., Carter, M., \& Sanli, E. (2011). Decreasing the proportion of self-control trials during the acquisition period does not compromise the learning advantages in a self-controlled context. Research Quarterly for Exercise and Sport, 82, 624-633.

Robertson, E. M. (2009). From creation to consolidation: A novel framework for memory processing. PLoS Biology, 7, e19. doi:10.1371/ journal.pbio.1000019

Robertson, E. M., Pascual-Leone, A., \& Miall, R. C. (2004). Current concepts in procedural consolidation. Nature Reviews Neuroscience, 5, 576-582. doi:10.1038/nrn1426

Sanli, E. A., Patterson, J. T., Bray, S. R., \& Lee, T. D. (2013). Understanding self-controlled motor learning protocols through the Self-Determination Theory. Frontiers in Psychology, 3, 611. doi:10.3389/fpsyg.2012.00611

Schmidt, R. A., \& Lee, T. D. (2011). Motor control and learning: A behavioral emphasis (5th ed.). Champaign: Human Kinetics.

Simons, D. J. (2014). The value of direct replication. Perspectives on Psychological Science, 9, 76-80. doi:10.1177/1745691613514755

Ste-Marie, D. M., Carter, M. J., Law, B., Vertes, K., \& Smith, V. (2016). Self-controlled learning benefits: Exploring the contributions of selfefficacy and intrinsic motivation via path analysis. Journal of Sport Sciences, 34, 1650-1656. doi:10.1080/02640414.2015.1130236

Ste-Marie, D. M., Vertes, K. A., Law, B., \& Rymal, A. M. (2013). Learner-controlled self-observation is advantageous for motor skill acquisition. Frontiers in Psychology, 3, 556. doi:10.3389/fpsyg. 2012.00556

Swinnen, S. P. (1988). Post-performance activities and skill learning. In O. G. Meijer \& K. Roth (Eds.), Complex motor behaviour: The motor-action controversy (pp. 315-338). Amterdam: Elsevier Science.

Wulf, G., \& Lewthwaite, R. (2016). Optimizing performance through intrinsic motivation and attention for learning: The OPTIMAL theory of motor learning. Psychonomic Bulletin \& Review, 23, 13821414. doi:10.3758/s13423-015-0999-9 